clear (but erroneous) history that BCG vaccination had been given. As the Heaf test was only grade two positive and the chest radiograph was normal, she was discharged, in accordance with the British Thoracic Society guidelines. ${ }^{1} \mathrm{Had}$ her true immune status been appreciated she would have had chemoprophylaxis. A single point in the history therefore made a considerable difference to her management.

In any case, BCG vaccination is known to afford incomplete protection (nil to $80 \%$ in different series ${ }^{2}$ ). It is possible that the development of a strongly positive Heaf test or chest radiographic changes would be delayed in patients only partially protected by BCG vaccine. Furthermore, the duration of protection after vaccination is unknown, and there is no good correlation between tuberculin hypersensitivity and immunity to tuberculosis. ${ }^{34}$ It therefore seems to us to be safer to repeat the Heaf test and the chest radiograph three months as well as six weeks after the last known contact in all apparently negative children whether or not they have had BCG vaccination rather than to discharge them immediately from further follow up. The additional inconvenience is trivial, and, at least in this child, it seems likely that such a policy would have resulted in the earlier initiation of treatment.

1 Subcommittee of the Joint Tuberculosis Committee of the British Thoracic Society. Control and prevention of tuberculosis in Britain: an updated code of practice. BMF 1990; 300:995-9.

2 Fine PEM, Rodrigues LC. Modern vaccines: mycobacterial diseases. Lancet 1990;335:1016-20.

3 Hart PD'A, Sutherland I, Thomas J. The immunity conferred by effective BCG and vole bacillus vaccines, in relation to individual variations in induced tuberculin sensitivity and to technical variations in the vaccines. Tubercle 1967; 48:201-10.

4 Comstock GW. Vaccination against tuberculosis: controlled trials of BCG vaccination by the US Public Health Service. International conference on the application of vaccines against viral, rickettsial and bacterial disease of man. New York: Pan American Health Organisation, 1971:378-81.

\title{
Indwelling cannula for insulin administration in diabetes mellitus
}

\author{
A M Long, I A Hughes
}

\begin{abstract}
Experience with an indwelling subcutaneous Teflon cannula for insulin delivery to 10 children with diabetes mellitus is described. There were no significant complications during a one year trial period. The device may particularly benefit children during the early phase after diagnosis and for those with true needle phobia.
\end{abstract}

Multiple insulin injections may lead to distress and poor compliance in diabetic children particularly those of preschool age. We report the use of an indwelling, subcutaneous Teflon cannula (Insuflon, Viggo*) to give insulin to newly diagnosed diabetics and children who require a brief period of frequent insulin injections to regain adequate metabolic control.

\section{Patients and methods}

METHODS

The 24 guage Teflon cannula with an inner lumen diameter of $0.4 \mathrm{~mm}$ and an external diameter of $0.6 \mathrm{~mm}$ is similar in design to conventional cannulae used for intravenous treatment in neonatal units (figure $\mathrm{A}$ ). The external end was sealed by a rubber membrane

${ }^{*}$ The Insuflon device is manufactured in the UK by ViggoSpectramed and distributed by Medistron Limited, 6 LawsonHunt Industrial Park, Brodbridge Heath, Horsham, West Sussex, RH12 3JR. which covers a conical injection port. The total dead space of injection port and cannula is $0.0075 \mathrm{ml}$; this is equivalent to 0.75 units of insulin (100 units $/ \mathrm{ml}$ ). A topical anaesthetic cream containing lignocaine and prilocaine (EMLA, Astra) was applied to the skin one hour before inserting the cannula. After cleansing with alcohol, a roll of skin on the abdomen was lifted to insert the cannula subcutaneously at an oblique angle using a $0.4 \mathrm{~mm}$ steel stylet which was then removed. The device was secured with an adhesive patch (figure B). The cannula patency was maintained by the residual volume of insulin in the dead space.

\section{PATIENTS}

Ten children aged 4 to 13 years were studied. Five children had newly presented with diabetes, of whom three had severe ketoacidosis and required intravenous fluids and insulin. The subcutaneous cannula was inserted later to give short acting insulin every six hours. The other two newly diagnosed diabetics were given multiple insulin injections for 48 hours via the cannula and then changed to twice daily injections.

Two newly diagnosed diabetics were referred because they had refused twice daily injections of insulin. A cannula was inserted in both children without difficulty and they quickly adjusted to having regular injections.

The cannula was used in a further three 


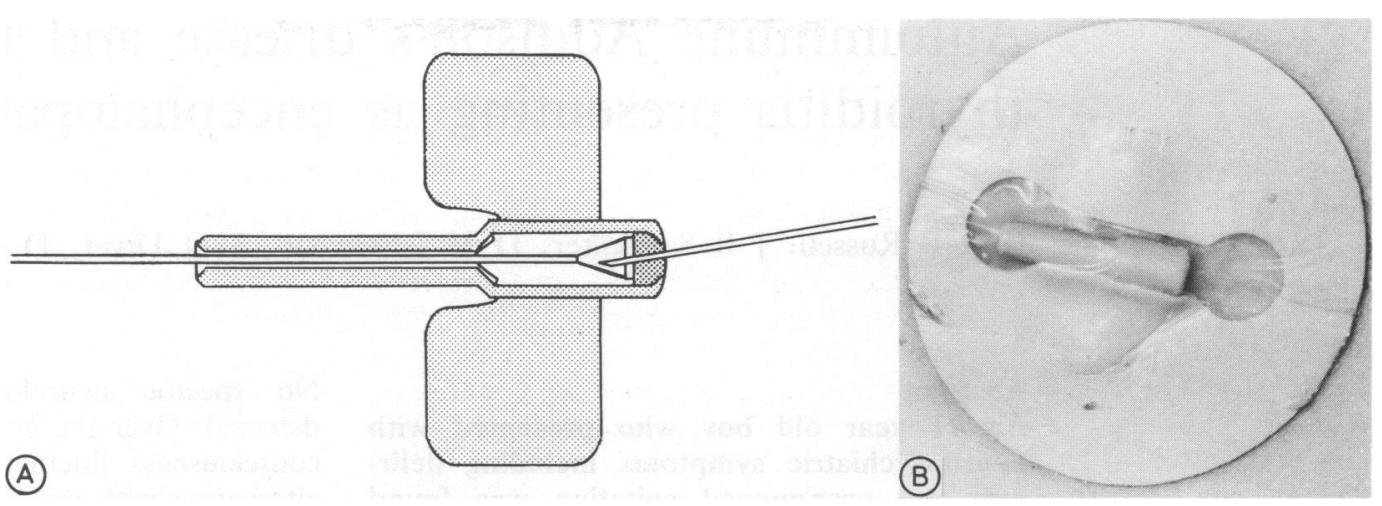

(A) Cross sectional diagram through cannula showing tip of needle in injection port. (B) Photograph of Insuflon in situ showing circular adhesive patch (tip of cannula to left of picture).

patients who needed a period of intensive insulin treatment during an intercurrent illness.

\section{Results}

The cannula was changed five days after discharge from hospital by a health visitor who was a specialist in diabetes. The parents continued giving insulin through the device twice daily and the period between change of site was adjusted for each patient. The mean duration in any one site was seven days, with a range of four to $\mathbf{1 0}$ days. All parents were able to replace the cannula themselves within two months of using this system.

Five patients continued to use the device at home for routine twice daily insulin injections over a period of three months to one year. Blood glucose profiles were comparable with other newly diagnosed diabetics and concentrations of glycated haemoglobin after three months, and in two cases, after six and nine months usage, were similar to those of other new diabetics attending our clinic.

At the end of the study period, two patients continued to use the device regularly, three patients having adapted to twice daily insulin injections without problem.

\section{COMPLICATIONS}

The only practical difficulty with use of the device was failure of the adhesive patch. Careful drying of the skin before application ensured that bathing, showering, swimming, and participation in energetic sports could be performed without loss of adhesion.

Minor complications included transient eczematous eruptions at the fixation site in two patients and the development of a small pustule at the insertion site in another patient. This followed prolonged use of the same site, the infection clearing once the cannula was resited.

Lipohypertrophy occurred in one patient who persistently used the same injection site. The problem quickly resolved when the cannula site was changed.

\section{Discussion}

Good metabolic control of diabetes leads to delayed onset, if not a lower incidence, of microvascular complications. ${ }^{1}$ These may also be delayed by good habits being established soon after diagnosis. ${ }^{2}$

Most diabetics will continue to require regular, injected insulin for the forseeable future. Devices that make this less unpleasant are likely to improve compliance and discourage resistance to multiple injection treatment. True needle phobia is most common during adolescence $^{3}$ and may be the result of unpleasant experiences in earlier life. ${ }^{4}$

Studies in children ${ }^{5}$ and adults ${ }^{6}$ have shown that the subcutaneous cannula is well tolerated by diabetics of all ages. Using the device for multiple insulin injections leads to better compliance and improved metabolic control. Minor complications were similar to those reported in this study and generally resolved when the cannula was resited.

While the subcutaneous indwelling cannula was well tolerated and liked by patients and parents for ease of use and convenience, the relatively high unit cost means that this mode of treatment is probably not appropriate for routine general use in the child with uncomplicated diabetes. Those with true needle phobia or persistent abuse of one injection site, however, would benefit from the Insuflon device. A period of pain free injections may provide an easier phase of adaptation for newly diagnosed children and their parents.

1 Leslie ND, Sperling MA. Relation of metabolic control to complications in diabetes mellitus. $\mathcal{J}$ Pediatr 1986;108: 491-7.

2 Weber B, Burger W, Hartmann R, et al. Risk factors for the development of retinopathy in children and adolescents with type 1 (insulin-dependent) diabetes mellitus. Diabetologia 1986;29:23-9.

3 Tattersall RB, Lowe J. Diabetes in adolescence. Diabetologia 1981;20:517-23.

4 Agras S, Sylvester D, Oliveau D. The epidemiology of common fears and phobia. Compr Psychiatry 1969;10: 151-6.

5 Hanas R, Ludvigsson J. Side effects and in-dwelling times of subcutaneous catheter for insulin injection: a new device for injecting insulin with a minimum of pain in the treatment of insulin-dependent diabetes mellitus. Diabetes Res Clin Pract 1990;10:73-83.

6 Rayman G, Wise PH. An indwelling subcutaneous FEP cannula for intermittent insulin injection: patient experience and effect on diabetic control. Diabetic Medicine 1988; 5:592-5. 